\title{
Role of the androgen signaling axis in genitourinary malignancies
}

\author{
Brian M. Shinder ${ }^{1}$, Adam Shupe ${ }^{1}$, Geun Taek Lee ${ }^{1}$, Mark N. Stein ${ }^{2}$, Isaac Y. Kim ${ }^{1}$, Eric A. Singer ${ }^{1}$ \\ ${ }^{1}$ Section of Urologic Oncology, Rutgers Cancer Institute of New Jersey and Rutgers Robert Wood Johnson Medical School, New Brunswick, NJ, \\ USA; ${ }^{2}$ Division of Medical Oncology, Rutgers Cancer Institute of New Jersey and Rutgers Robert Wood Johnson Medical School, New Brunswick, \\ NJ, USA \\ Contributions: (I) Conception and design: BM Shinder, A Shupe, EA Singer; (II) Administrative support: EA Singer; (III): Provision of study materials \\ or patients: None; (IV): Collection and assembly of data: All authors; (V): Data analysis and interpretation: All authors; (VI) Manuscript writing: All \\ authors; (VII) Final approval of manuscript: All authors. \\ Correspondence to: Eric A. Singer, MD, MA, FACS. Assistant Professor of Surgery, Director, Kidney Cancer Program, Section of Urologic Oncology, \\ Rutgers Cancer Institute of New Jersey, 195 Little Albany Street Room 4563, New Brunswick, NJ 08903, USA. Email: eric.singer@rutgers.edu.
}

\begin{abstract}
As we learn more about the molecular biology of genitourinary malignancies, novel therapeutic strategies can be developed. This is especially crucial for prostate, renal, and bladder cancer, where mortality rates remain high especially in advanced disease states. The androgen signaling axis and the androgen receptor (AR) are areas that are actively being explored for their role in these diseases. Although long been associated with prostate cancer development and progression, the role of AR in renal cell carcinoma (RCC) and bladder cancer is becoming recognized as well. This review will highlight the current research into the role of the androgen signaling axis in genitourinary malignancies and how this pathway is being used to expand our therapeutic armamentarium.
\end{abstract}

Keywords: Androgen receptor (AR); prostate cancer; bladder cancer; kidney cancer

Submitted Feb 21, 2018. Accepted for publication Mar 12, 2018.

doi: $10.21037 /$ tcr.2018.03.41

View this article at: http://dx.doi.org/10.21037/tcr.2018.03.41

\section{Introduction}

Genitourinary malignancies continue to pose a significant health burden on both men and women worldwide. In 2018 prostate, kidney, and urinary bladder cancers are estimated to be responsible for over $31 \%$ of all new cancer cases in men with 315,040 estimated new cases of prostate and urinary system cancers in men and women combined (1). Accordingly, the therapeutic strategies for genitourinary cancers must continue to evolve. Contemporary systemic treatment options include targeted therapies, immunotherapies, cytotoxic chemotherapy, and endocrine-based approaches, with much of the current research focused on finding novel treatment targets and manipulating known biological pathways in more innovative ways. One such area of active investigation involves the androgen signaling axis, which is comprised of the androgen receptor (AR) and androgen synthesis pathways.

While established as a suitable target for prostate cancer development and progression for over 70 years, the androgen signaling axis is now being exploited for the other genitourinary cancers as well. For example, the AR is a nuclear receptor that mediates gene transcription through the binding of various androgens and their effect on cell proliferation and differentiation make them an intriguing target for therapy. This review will highlight the current research regarding such therapies for prostate, bladder, and kidney cancer.

\section{Androgen signaling in prostate cancer}

A seminal moment in prostate cancer research occurred when Huggins and Hodges at the University of Chicago in the early 1940s first realized that prostate cancer development and metastasis was directly related to androgen signaling (2). This Nobel-prize winning discovery has led to the development of a multitude of pharmacological agents designed to disrupt the gonadal androgen synthesis pathway 


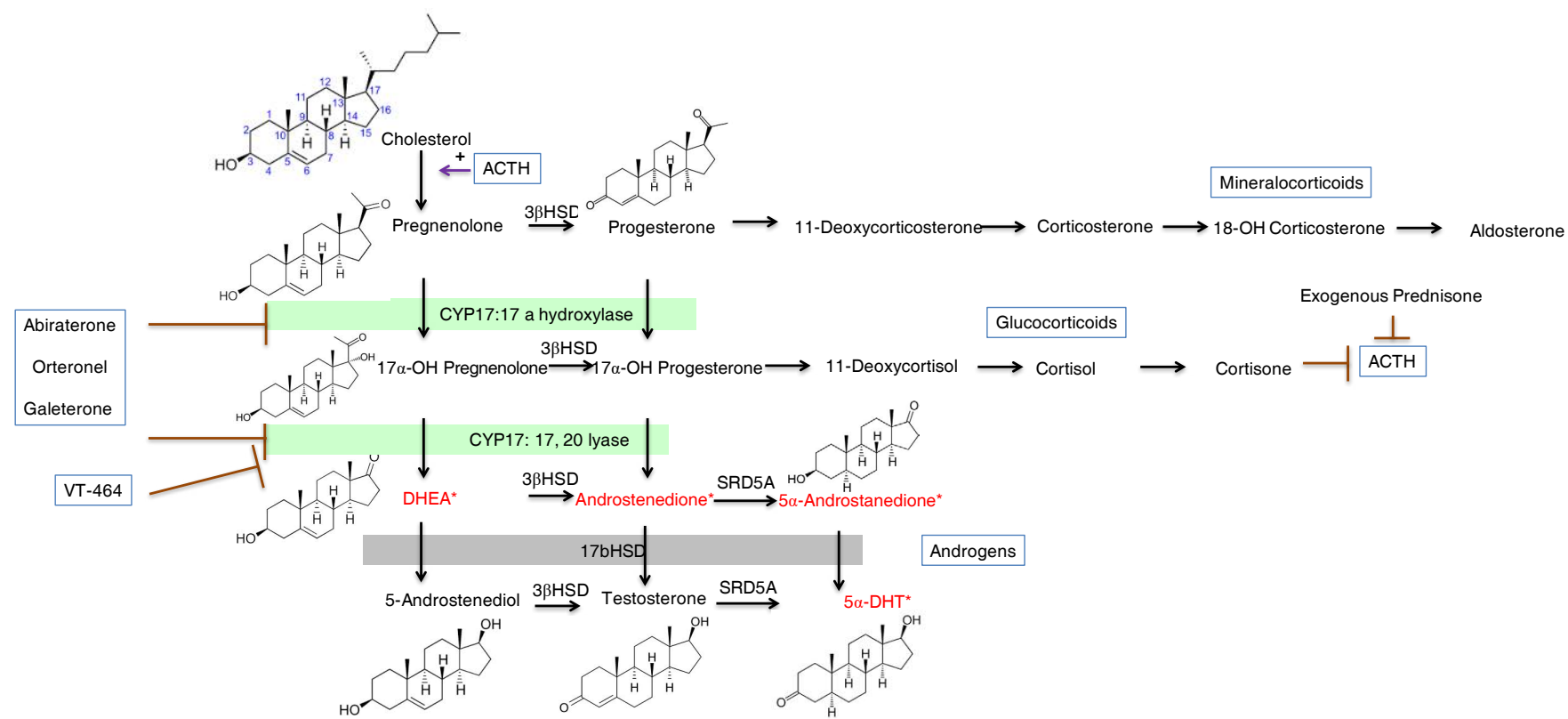

Figure 1 Steroid synthesis pathway. Mineralocorticoid, glucocorticoid, dehydroepiandrosterone, and androstenediol synthesis take place in the adrenal gland. Testosterone is converted to DHT in peripheral tissue. Abiraterone inhibits both the $17 \alpha$-hydroxylase and 17,20-lyase activity of the cytochrome p450 enzyme CYP17. Orteronel and galeterone have increased specificity for 17,20-lyase relative to $17 \alpha$-hydroxylase. VT-464 has 10 -fold in vitro specificity for the 17,20-lyase reaction over $17 \alpha$-hydroxylase. Androgens in the

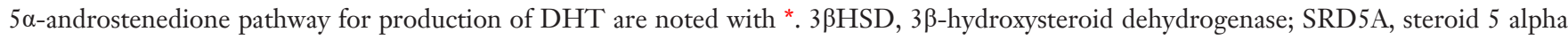
reductase; DHT, dihydrotestosterone; DHEA, dehydroepiandrosterone. Reproduced with permission from (3).

\section{(Figure 1) (3).}

Traditionally, androgen deprivation therapy (ADT) has been utilized as first-line treatment in men with metastatic disease. In this setting, it provides important palliative benefits such as reducing bone pain, pathologic fractures, and renal failure (4). Though it has demonstrated efficacy in delaying prostate cancer death, there is limited evidence that ADT improves overall survival $(5,6)$. Most men receive multiple forms of ADT across several lines of therapy $(7,8)$. However, nearly all of these men progress to the hormonerefractory state of castrate-resistant prostate cancer (CRPC), which inevitably results in death. The initial treatment response to ADT is limited, as tumor cells evolve complicated mechanisms to reactivate the AR signaling axis, including AR amplification, gene mutations, splice- variant pathways, and aberrant co-regulator activities $(9,10)$. A thorough understanding of the androgen signaling axis led to the development of second-line hormonal therapies that exploit this continued influence of androgen, even in the castrate-state (7).

CYP17 is a fundamental component in the synthesis of non-gonadal androgens from cholesterol, catalyzing two key reactions involved in this production of sex steroids (11). Ketoconazole, a weak inhibitor of CYP17, is often enlisted as an off-label therapy for CRPC. Its use in prostate cancer treatment showed a decrease in serum prostate-specific antigen (PSA) by $\geq 50 \%$ in $27 \%$ of patients diagnosed with CRPC (12). Following these findings, abiraterone was developed as the next generation inhibitor of CYP17. Abiraterone irreversibly binds to the CYP17 enzyme and has proven to be 10-30 times more potent in the inhibition of CYP17 compared to ketoconazole $(3,13)$. De Bono et al. reported on a phase III trial of abiraterone in men with PCa and found a significant improvement in overall survival compared to placebo [14.8 vs. 10.9 months; hazard ratio $(\mathrm{HR})=0.65 ; 95 \%$ confidence interval $(\mathrm{CI})$, 0.54-0.77; $\mathrm{P}<0.001]$ as well as improvements in time to PSA progression, progression-free survival, and PSA response rate (14).

A second-generation CYP17 pharmaceutical agent known as orteronel has been developed which has both $17 \alpha$-hydroxylase and 17,20-lyase activity. The $17 \alpha$-hydroxylase activity is a potent inhibitor of CYP17, while the 17,20-lyase activity is used to counteract the 
excess mineralocorticoid synthesis usually associated with the inhibition of CYP17 (15). The results from the phase I/II have shown that orteronel is a more specific CYP17 inhibitor that has fewer serious side effects when compared to abiraterone. Data is still being collected from larger phase III studies that could help push orteronel into routine clinical practice. Additionally, the molecules VT-464 and galeterone are both pharmaceutical agents used to specifically target the 17,20-lyase reaction over $17 \alpha$-hydroxylase activity. In contrast to abiraterone and orteronel, these newly described agents have an increased specificity towards 17,20-lyase, which results in the inhibition of testosterone synthesis while causing only minimal changes to patient cortisol levels (16).

Further pharmaceutical agents involved in the direct inhibition of AR signaling in an effort to lower the overall increased AR activity often seen in CRPC have also been developed.

Enzalutamide is an AR signaling inhibitor with a high affinity for the AR that is used to dampen such signaling activity. When enzalutamide binds to the AR receptor, it down regulates its signaling by inhibiting nuclear translocation of the AR, DNA binding, and coactivator recruitment (17). Scher et al. reported on the AFFIRM clinical trial, a phase III investigation showing that the median overall survival increased by 4.8 months in men with CRPC who received enzalutamide compared to placebo (18.4 vs. 13.6 months, respectively; $\mathrm{P}<0.001)(17)$. A molecule with a similar mechanism of action, apalutamide, tested in the SPARTAN trial where in combination with ADT, men with non-metastatic CRPC had a median free survival of 40.5 months compared to 16.2 months in the placebo + ADT group (HR $=0.45 ; 95 \%$ CI, 0.32-0.63; $\mathrm{P}<0.001)(18)$. FDA approval for apalutamide was recently granted for the treatment of this population of men.

Novel androgen independent therapies such as chemotherapy or immunotherapy have been employed to curtail further disease progression and improve survival. Traditionally, these therapies are delayed until castrationresistant disease is documented. However, the results of STAMPEDE and CHAARTED have begun to shift this treatment paradigm. Reporting on the CHAARTED study, Sweeney et al. showed a 13.6-month increase in overall survival when docetaxel was given at the initiation of ADT compared to ADT alone (57.6 vs. 44.0 months; $\mathrm{HR}=0.61$; $95 \%$ CI, 0.47-0.80; $\mathrm{P}<0.0001)$ in men with metastatic prostate cancer (19). In the STAMPEDE trial, patients lived longer across all study groups, though the effect of early docetaxel treatment was still seen (81 vs. 71 months; $\mathrm{HR}=0.78 ; 95 \%$ CI, 0.66-0.93; $\mathrm{P}=0.006)$ (20).

Most recently, "deep androgen suppression" has been investigated, with the goal of disrupting the androgen signaling via different mechanisms simultaneously in order to maximize the effectiveness of therapy. For example, the LATITUDE trial randomly assigned 1,199 men with newly diagnosed high-risk, metastatic, castration sensitive prostate cancer (CSPC) to receive abiraterone plus ADT and prednisone, or ADT and placebo (21). The authors found that after a median follow-up of 30.4 months, the relative risk of death was $38 \%$ lower in the abiraterone plus $\mathrm{ADT}$ group ( $\mathrm{HR}=0.62 ; 95 \% \mathrm{CI}, 0.51-0.76 ; \mathrm{P}<0.001)$. Based on the results of this trial abiraterone + prednisone is now FDA approved for the treatment of CSPC. Testing the effects of ADT + abiraterone, an abiraterone branch was opened in the STAMPEDE trial in 2011, where 960 patients had abiraterone added to their standard therapy regimens. Patients who received this added therapy experienced a $37 \%$ improvement in overall survival and $71 \%$ improvement in FFS (20).

Though these newly designed targeted therapies have certainly proved to be beneficial, a subset of men will still develop resistance to these agents. One such mechanism of resistance to enzalutamide and abiraterone is a result of AR splice variants, specifically AR-V7. AR-V7 positive patients experienced no clinical benefit when treated with enzalutamide or abiraterone, suggesting the presence of drug resistance (22). Although this link between therapeutic resistance and AR-V7 had been revealed, the underlying mechanisms behind such findings have not been fully elucidated.

There is much work ongoing to characterize the different resistance pathways to AR targeting drugs in prostate cancer. Although many of these drugs have been established to be beneficial, additional resistance pathways should be more thoroughly mapped out in order to improve patient selection for AR directed therapies.

\section{Androgen signaling in bladder cancer}

Bladder cancer remains a difficult disease process to treat, as patients with non-muscle invasive disease are subject to multiple cystoscopies and often repeat endoscopic resections (23). In patients with muscle invasive disease, treatment is highly morbid and patients must choose between chemotherapy plus external beam radiation or radical cystectomy/urinary diversion with or without 
neoadjuvant chemotherapy (24). Thus, novel therapeutic strategies are needed, especially when traditional treatment pathways are no longer effective. Recent evidence has suggested that the AR signaling pathway plays a role in bladder cancer development and progression, leading some to investigate its potential as a therapeutic target.

Epidemiologic data strongly supports the idea that men are at an increased risk of developing bladder cancer compared to women, although women have been shown to present with more advanced disease. Data collected by Dobruch et al. from 1998-2008 showed a universal increase in the overall number of bladder cancer cases in both men and women, however, men displayed $25 \%$ faster rate of increase when compared to women (25). Some have proposed that this gender difference in bladder cancer rates can be attributed to a larger percentage of men who used to smoke than women. However, this gender difference was shown in various studies to be independent of previous smoking status $(25,26)$. To explain this disparity in rates of bladder cancer between males and females, hormonal factors have been postulated to play a role. Ding et al. showed a correlation between male bladder cancer proliferation/migration and $\mathrm{AR}$ expression in two different bladder cancer cell lines (27). In UM-UC-3 cells in which $\mathrm{AR}$ is overexpressed, proliferation/migration was suppressed when AR was silenced. On the other hand, T24 cells in which had low levels of AR expression, proliferation and migration was significantly increased by AR overexpression. Furthermore, AR overexpression was associated with higher VEGF and CD24 expression, which have been previously been implicated in bladder cancer progression $(28,29)$.

Kameyama et al. carried out a similar study on the T24 cells, investigating the role of AR in gemcitabine-resistant bladder cancer cells, which is commonly used to treat urothelial carcinoma, as well as the effects enzalutamide would have on this progressive disease. Microarray analysis showed an overall upregulation of AR expression in T24 cell lines that were resistant to gemcitabine (T24GR), when compared to normal T24 cell lines, suggesting that AR upregulation may be an important mechanism in bladder cancer chemoresistance (30). With this in mind, enzalutamide was used to investigate the effects an AR inhibitor would have on these cancer cells. Enzalutamide proved to cause cell cycle arrest in T24GR cell lines, resulting in the inhibition of T24GR cell growth and reduction in AR transcriptional activity (30).

Izumi et al. sought to further investigate the clinical significance of AR blockade on patients with bladder cancer.
They evaluated 162 men with bladder cancer from a cohort of men who had also been diagnosed with prostate cancer between 1991-2013. With a median follow up time of 62 months, the data revealed a $50 \%$ recurrence rate in patients who were not subjected to ADT and a $22 \%$ recurrence rate in patients received $\mathrm{ADT}$ (5-year actuarial recurrence-free survival: $76 \%$ vs. $40 \%$; $\mathrm{P}<0.001)(31)$. Similarly, Shiota et al. carried out a study with the similar goal of determining the effect ADT on intravesical recurrence rates for non-muscle invasive bladder cancer. The study involved 228 men (32 with and 196 without ADT), with a median follow up of 3.6 and 3 years, respectively. Recurrence was shown to develop in $30.1 \%$ of men who were not treated with ADT compared to $12.5 \%$ for men who received ADT. In addition, tumor progression to muscle invasive bladder cancer was seen in $3.1 \%$ of men who were not exposed to ADT, while none of the men who were received ADT progressed (32).

Other agents that influence the androgen-signaling axis have been studied in order to determine their ability to suppress bladder cancer. Using data from the Prostate, Lung, Colorectal, and Ovarian (PLCO) cancer screening trial, Morales et al. evaluated the role that finasteride, a 5 -alpha reductase inhibitor, had on the suppression of bladder cancer progression (33). A multiple cox regression analysis linked the use of finasteride with a significant decrease in bladder cancer development $(\mathrm{HR}=0.634 ; 95 \%$ CI, 0.493-0.816; $\mathrm{P}=0.0004$ ) providing further evidence that manipulation of the androgen axis may have a role in the treatment of patients with bladder cancer (33).

\section{Androgen signaling in renal cell carcinoma (RCC)}

Current systemic therapeutic regimens for advanced and metastatic RCC are still predominantly based on targeted therapies (34). While benefits in survival and disease progression have been shown, complete responses are rarely observed. As combination treatments with targeted therapies are often highly toxic, newer treatment options are needed (34-37). A growing body of evidence indicates that the androgen signaling axis could serve in such a manner.

Ha et al. examined renal tissue from 115 patients with primary pathological stage T1 or T2 RCC and compared them to 57 non-malignant controls (38). They found no significant difference in AR mRNA expression in the normal tissue samples when compared to the AR expression in tumor tissue samples $(\mathrm{P}=0.684)$. However, $\mathrm{AR}$ levels 

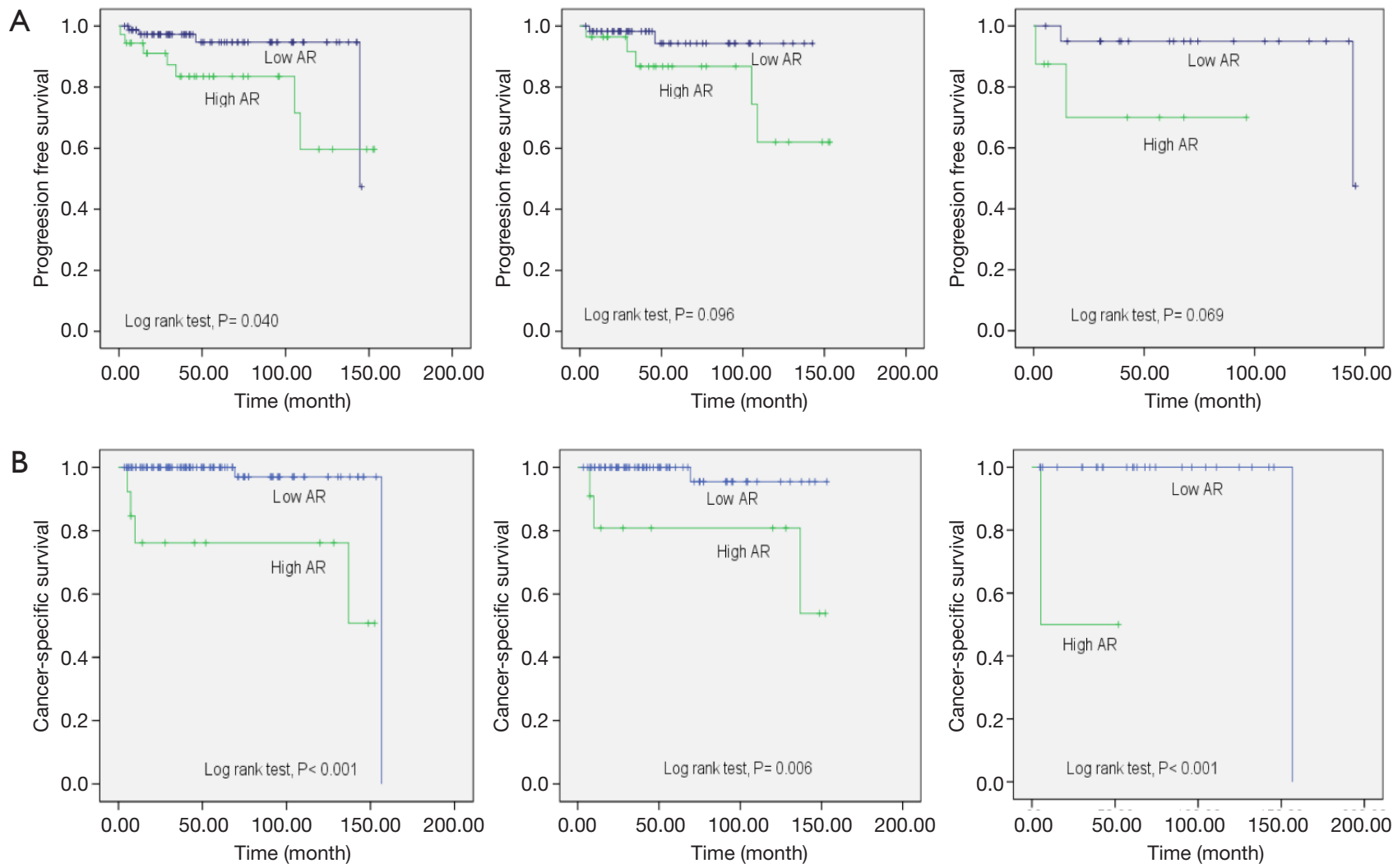

Figure 2 Kaplan-Meier curves of estimated progression-free (A) and cancer specific (B) survival by AR mRNA expression levels. Reproduced with permission from (38). AR, androgen receptor.

were shown to be significantly higher in patients with pT2 tumors than in those with pT1 tumors. The authors also showed that, on average, patients with a higher AR expression levels had shorter progression free survival ( $\log$ rank test $\mathrm{P}=0.040$, Figure $2 A$ ) and lower overall survival rates (log rank test $\mathrm{P}=0.040$, Figure $2 B$ ), linking AR expression to poor prognosis. Importantly, $25.2 \%$ of subjects in this study were women, indicating that this is not a gender-driven phenomenon and that agents targeting this pathway may have a role regardless of the patient's sex.

Similarly, He et al. transfected normal human kidney epithelial cells with AR and showed that these cells quantitatively formed a larger and a higher number of colonies in soft agar when transformed by a carcinogen (39). The addition of functional AR in stable RCC tumor cell lines increased cellular proliferation compared to their vector controls, and when enzalutamide was added, these effects were suppressed. Analogous findings have been shown with an in vivo model as well. When AR-positive Caki2 RCC cell lines were orthotopically xenografted into nude mice, the tumor growth rate was significantly decreased when mice underwent castration compared to when the mice were not castrated (40). Furthermore, male mice with these Caki2 xenografts were exposed to enzalutamide or abiraterone acetate. After three weeks of treatment, tumor volume was twenty- and nine-fold smaller in the enzalutamide and abiraterone groups, respectively, compared to the controls (Figure 3) (40), Taken together, these results suggest that AR expression may help initiate RCC tumor growth and support RCC progression.

Other studies have attempted to elucidate the mechanism of AR involvement in RCC. Guan et al. showed that AR acts in part to mediate the AKT $\rightarrow \mathrm{NF}-\kappa \mathrm{B} \rightarrow$ CXCL5 signaling pathway (41). This PI3K/AKT pathway, which is a target of mTOR inhibitors and is involved in endothelial cell recruitment, is now well known to be closely associated with RCC progression. Thus, AR mediated modulation of the pathway may influence the tumor microenvironment via increased endothelial cell recruitment and promoting angiogenesis. Additionally, in vivo studies were carried out 

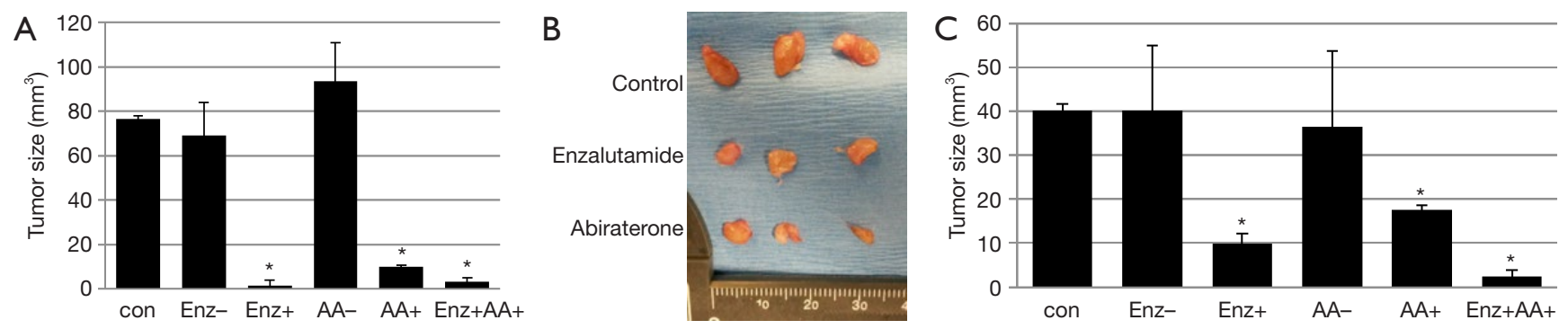

Figure 3 Targeting the androgen signaling axis decreases tumor growth in AR-positive human RCC cell line, Caki2, xenografts. (A) After inoculating the flanks with Caki2, 30 mice were randomized into six groups of five mice each. The treatment was rendered as indicated. Both enzalutamide (Enz) and abiraterone acetate (AA) decreased tumor volume dramatically. (B) Gross picture of tumors collected from mice after treatment. (C) After establishing the tumors, 30 mice were surgically castrated and treated with the indicated agents. Again, Enz and Abi decreased tumor volume significantly. *, statistically significant. Reproduced with permission from (40).

on nude mice models in order to determine if a similar trend would be observed. AR negative and positive cell lines were orthotopically xenografted into the kidney capsules of nude mice. Interestingly, those mice injected with the AR positive cells had larger tumor sizes in addition to more endothelial cell recruitment (41).

These reports not only exemplify the androgen signaling axis' potential importance in RCC progression, but also propose a mechanism on how this effect is carried out. Building on the results from such studies, clinical trials have been developed in an attempt to see how these possible therapeutic agents targeting the AR effects RCC in a clinical setting. The BARE trial (Blockade of Androgens in Renal cell carcinoma using Enzalutamide), a phase 0 clinical trial (NCT02885649), aims to uncover the effects of enzalutamide on tumor growth prior to surgical resection. Patients who have a clinical T1N0M0 renal mass that is biopsy-proven to be clear cell RCC with AR expression of $\geq 4,580$ copies $/ \mu$ g RNA are eligible to participate. Enrolled subjects will receive enzalutamide for 4 weeks and then undergo either partial or radical nephrectomy. Cell proliferation and tumor apoptosis as measured by annexin will be used as the primary outcome measures of this study. The BARE trial may offer new insights on the function of $\mathrm{AR}$ and its role as a therapeutic target in RCC.

\section{Conclusions}

The treatment landscape of genitourinary malignancies continues to evolve as our understanding of the genomic and molecular biology of these diseases is advanced. Current therapeutic modalities for these disease processes include targeted therapies, cytotoxic chemotherapies, and immuno-oncologic agents. Although robust responses can sometimes be seen, objective response rates are often limited and treatment options are usually less efficacious for those who progress after initial therapy. Approaches utilizing a combination of therapies are appealing but are frequently limited by their increased toxicity profiles. In this respect, targeting the AR and the androgen synthesis pathway may offer a novel approach to inhibiting disease progression and improving patient outcomes. While the androgen signaling axis has been known to be an important driving force behind prostate cancer development for some time, it has recently been implicated in both bladder cancer and RCC as well. Thus, further work to elucidate the complete mechanisms of such findings is imperative.

\section{Acknowledgments}

Funding: This work is supported by a grant from the National Cancer Institute (P30CA072720).

\section{Footnote}

Conflicts of Interest: Dr. EA Singer and Dr. IY Kim receive research funding from Astellas/Medivation for the BARE trial. The other authors have no conflicts of interest to declare.

Ethical Statement: The authors are accountable for all aspects of the work in ensuring that questions related to the accuracy or integrity of any part of the work are appropriately investigated and resolved.

Open Access Statement: This is an Open Access article 
distributed in accordance with the Creative Commons Attribution-NonCommercial-NoDerivs 4.0 International License (CC BY-NC-ND 4.0), which permits the noncommercial replication and distribution of the article with the strict proviso that no changes or edits are made and the original work is properly cited (including links to both the formal publication through the relevant DOI and the license). See: https://creativecommons.org/licenses/by-nc-nd/4.0/.

\section{References}

1. Siegel RL, Miller KD, Jemal A. Cancer statistics, 2018. CA Cancer J Clin 2018;68:7-30.

2. Huggins C, Hodges CV. Studies on prostatic cancer. I. The effect of castration, of estrogen and of androgen injection on serum phosphatases in metastatic carcinoma of the prostate. 1941. J Urol 2002;167:948-51; discussion 52.

3. Stein MN, Patel N, Bershadskiy A, et al. Androgen synthesis inhibitors in the treatment of castration-resistant prostate cancer. Asian J Androl 2014;16:387-400.

4. Pagliarulo V, Bracarda S, Eisenberger MA, et al. Contemporary role of androgen deprivation therapy for prostate cancer. Eur Urol 2012;61:11-25.

5. Sharifi N, Gulley JL, Dahut WL. Androgen deprivation therapy for prostate cancer. JAMA 2005;294:238-44.

6. Nair B, Wilt T, MacDonald R, et al. Early versus deferred androgen suppression in the treatment of advanced prostatic cancer. Cochrane Database Syst Rev 2002:CD003506.

7. Virgo KS, Basch E, Loblaw DA, et al. Second-Line Hormonal Therapy for Men With Chemotherapy-Naive, Castration-Resistant Prostate Cancer: American Society of Clinical Oncology Provisional Clinical Opinion. J Clin Oncol 2017;35:1952-64.

8. Singer EA, Golijanin DJ, Miyamoto H, et al. Androgen deprivation therapy for prostate cancer. Expert Opin Pharmacother 2008;9:211-28.

9. Karantanos T, Corn PG, Thompson TC. Prostate cancer progression after androgen deprivation therapy: mechanisms of castrate resistance and novel therapeutic approaches. Oncogene 2013;32:5501-11.

10. Polotti CF, Kim CJ, Chuchvara N, et al. Androgen deprivation therapy for the treatment of prostate cancer: a focus on pharmacokinetics. Expert Opin Drug Metab Toxicol 2017;13:1265-73.

11. Vasaitis TS, Bruno RD, Njar VC. CYP17 inhibitors for prostate cancer therapy. J Steroid Biochem Mol Biol 2011;125:23-31.
12. Small EJ, Halabi S, Dawson NA, et al. Antiandrogen withdrawal alone or in combination with ketoconazole in androgen-independent prostate cancer patients: a phase III trial (CALGB 9583). J Clin Oncol 2004;22:1025-33.

13. Chan FC, Potter GA, Barrie SE, et al. 3- and 4-pyridylalkyl adamantanecarboxylates: inhibitors of human cytochrome P450(17 alpha) (17 alpha-hydroxylase/C17,20-lyase). Potential nonsteroidal agents for the treatment of prostatic cancer. J Med Chem 1996;39:3319-23.

14. de Bono JS, Logothetis CJ, Molina A, et al. Abiraterone and increased survival in metastatic prostate cancer. $\mathrm{N}$ Engl J Med 2011;364:1995-2005.

15. Yamaoka M, Hara T, Kusaka M. Overcoming persistent dependency on androgen signaling after progression to castration-resistant prostate cancer. Clin Cancer Res 2010;16:4319-24.

16. Bird IM, Abbott DH. The hunt for a selective 17,20 lyase inhibitor; learning lessons from nature. J Steroid Biochem Mol Biol 2016;163:136-46.

17. Scher HI, Fizazi K, Saad F, et al. Increased survival with enzalutamide in prostate cancer after chemotherapy. $\mathrm{N}$ Engl J Med 2012;367:1187-97.

18. Smith MR, Saad F, Chowdhury S, et al. Apalutamide Treatment and Metastasis-free Survival in Prostate Cancer. N Engl J Med 2018.

19. Sweeney CJ, Chen YH, Carducci M, et al. Chemohormonal Therapy in Metastatic Hormone-Sensitive Prostate Cancer. N Engl J Med 2015;373:737-46.

20. James ND, Sydes MR, Clarke NW, et al. Addition of docetaxel, zoledronic acid, or both to first-line longterm hormone therapy in prostate cancer (STAMPEDE): survival results from an adaptive, multiarm, multistage, platform randomised controlled trial. Lancet 2016;387:1163-77.

21. Fizazi K, Tran N, Fein L, et al. Abiraterone plus Prednisone in Metastatic, Castration-Sensitive Prostate Cancer. N Engl J Med 2017;377:352-60.

22. Antonarakis ES, Lu C, Wang H, et al. AR-V7 and resistance to enzalutamide and abiraterone in prostate cancer. N Engl J Med 2014;371:1028-38.

23. Noyes K, Singer EA, Messing EM. Healthcare economics of bladder cancer: cost-enhancing and cost-reducing factors. Curr Opin Urol 2008;18:533-9.

24. Novara G, Catto JW, Wilson T, et al. Systematic review and cumulative analysis of perioperative outcomes and complications after robot-assisted radical cystectomy. Eur Urol 2015;67:376-401.

25. Dobruch J, Daneshmand S, Fisch M, et al. Gender and 
Bladder Cancer: A Collaborative Review of Etiology, Biology, and Outcomes. Eur Urol 2016;69:300-10.

26. Krabbe LM, Svatek RS, Shariat SF, et al. Bladder cancer risk: Use of the PLCO and NLST to identify a suitable screening cohort. Urol Oncol 2015;33:65 e19-25.

27. Ding G, Yu S, Cheng S, et al. Androgen receptor (AR) promotes male bladder cancer cell proliferation and migration via regulating CD24 and VEGF. Am J Transl Res 2016;8:578-87.

28. Yang CC, Chu KC, Yeh WM. The expression of vascular endothelial growth factor in transitional cell carcinoma of urinary bladder is correlated with cancer progression. Urol Oncol 2004;22:1-6.

29. Hofner T, Macher-Goeppinger S, Klein C, et al. Expression and prognostic significance of cancer stem cell markers CD24 and CD44 in urothelial bladder cancer xenografts and patients undergoing radical cystectomy. Urol Oncol 2014;32:678-86.

30. Kameyama K, Horie K, Mizutani K, et al. Enzalutamide inhibits proliferation of gemcitabine-resistant bladder cancer cells with increased androgen receptor expression. Int J Oncol 2017;50:75-84.

31. Izumi K, Taguri M, Miyamoto H, et al. Androgen deprivation therapy prevents bladder cancer recurrence. Oncotarget 2014;5:12665-74.

32. Shiota M, Kiyoshima K, Yokomizo A, et al. Suppressed Recurrent Bladder Cancer after Androgen Suppression with Androgen Deprivation Therapy or 5alpha-Reductase Inhibitor. J Urol 2017;197:308-13.

33. Morales EE, Grill S, Svatek RS, et al. Finasteride Reduces Risk of Bladder Cancer in a Large Prospective Screening Study. Eur Urol 2016;69:407-10.

34. Modi PK, Farber NJ, Singer EA. Precision Oncology: Identifying Predictive Biomarkers for the Treatment of

Cite this article as: Shinder BM, Shupe A, Lee GT, Stein MN, Kim IY, Singer EA. Role of the androgen signaling axis in genitourinary malignancies. Transl Cancer Res 2018;7(4):11351142. doi: $10.21037 /$ tcr.2018.03.41
Metastatic Renal Cell Carcinoma. Transl Cancer Res 2016;5:S76-80.

35. Barrisford GW, Singer EA, Rosner IL, et al. Familial renal cancer: molecular genetics and surgical management. Int J Surg Oncol 2011;2011:658767.

36. Allard CB, Gelpi-Hammerschmidt F, Harshman LC, et al. Contemporary trends in high-dose interleukin-2 use for metastatic renal cell carcinoma in the United States. Urol Oncol 2015;33:496 e11-6.

37. Flaherty KT, Manola JB, Pins M, et al. BEST: A Randomized Phase II Study of Vascular Endothelial Growth Factor, RAF Kinase, and Mammalian Target of Rapamycin Combination Targeted Therapy With Bevacizumab, Sorafenib, and Temsirolimus in Advanced Renal Cell Carcinoma--A Trial of the ECOGACRIN Cancer Research Group (E2804). J Clin Oncol 2015;33:2384-91.

38. Ha YS, Lee GT, Modi P, et al. Increased Expression of Androgen Receptor mRNA in Human Renal Cell Carcinoma Cells is Associated with Poor Prognosis in Patients with Localized Renal Cell Carcinoma. J Urol 2015;194:1441-8.

39. He D, Li L, Zhu G, et al. ASC-J9 suppresses renal cell carcinoma progression by targeting an androgen receptordependent HIF2alpha/VEGF signaling pathway. Cancer Res 2014;74:4420-30.

40. Lee GT, Han CS, Kwon YS, et al. Intracrine androgen biosynthesis in renal cell carcinoma. Br J Cancer 2017;116:937-43.

41. Guan Z, Li C, Fan J, et al. Androgen receptor (AR) signaling promotes RCC progression via increased endothelial cell proliferation and recruitment by modulating AKT --> NF-kappaB --> CXCL5 signaling. Sci Rep 2016;6:37085. 